\title{
RUNWAY INCURSIONS IN NORWAY 2009-2019: A CASE STUDY OF EXTENDED INVESTIGATION REPORTS
}

\author{
Jarle EID', Preben B. JENSEN ${ }^{2}$ \\ 1 Center for Crisis Psychology, University of Bergen, Norway \\ 2 Flesland Air traffic Control, Avinor Air Navigation Services, Norway
}

Source of support: The research has received no external funding and the authors have not observed any conflict of interest regarding this study.

Author's address: J. Eid, University of Bergen, Møllendalsbakken 9, 5020 Bergen, Norway, Tel.: +47 55589188, e-mail: Jarle. Eid@uib.no

Abstract: The aim of the present study is to examine case studies from extended investigations of runway incursions in Norway from 2009-2019. Runway incursions involves an incorrect presence of an aircraft, vehicle or person on the runway and represents a relatively frequent threat to safety in the aviation industry. A content analysis and classification of the extended investigation reports $(\mathrm{N}=7)$ revealed 42 explanatory factors that could be condensed into four categories related to perception, procedural errors, memory and decision-making. When mapped onto the theoretical framework of situational awareness about $70 \%$ of the explanatory factors were related to misperception of information, improper comprehension of information or incorrect projection of future actions, respectively. The present study suggests that situational awareness can serve as a useful theoretical framework to identify deficits in human factors associated with runway incursion incidents. The results from this study contributes to inform investigation into aviation hazards and training of air traffic controllers.

Keywords: air traffic control, aviation safety, human factors, situational awareness, runway incursion 


\section{INTRODUCTION}

The runway is one of the most safety-critical areas of an airport. Every day, there are at least two cases of unauthorized traffic on runways at airports within the European area [8]. A vehicle or an aircraft may mistakenly be permitted to travel on a busy runway or a vehicle or an aircraft may enter the runway without permission. Such events are called "runway incursion" and represents a significant hazard and threat to aviation safety. According to the International Civil Aviation Organization a runway incursion is a safety-critical incident involving the incorrect presence of an aircraft, vehicle, or person on the runway [13]. An important part of Air Traffic Control is therefore to detect and prevent runway incursion from taking place [8].

The objectives of air traffic control are to prevent collisions between aircraft, and between aircraft and vehicles, on the maneuvering area, and to keep an expeditious and orderly flow of air traffic [14]. Air traffic control is also expected to provide necessary information to pilots to conduct a safe flight and notify appropriate authority about aircraft in need of search and rescue services. To this end, they need to have a comprehensive "situation awareness" (SA) of current and upcom- ing events at the airport [30]. SA can be defined as "a person's perception of the elements in the environment within a volume of time and space, understanding the meaning of the elements, and predicting the status of the elements in the near future" [17]. Understanding situational awareness in complex application domains such as air traffic control will contribute to address crucial aspects of distributed cognitive processes in human machine systems [23]. For example, to achieve SA, the air traffic controller is dependent on inter alia monitoring systems such as air-to-ground communication and surveillance displays. To this end, decision support and runway incursion prevention systems have been introduced to lower the risk of human error [18]. The air traffic controller must, in addition to monitor multiple systems, visually monitor the area of responsibility, and operate "flight progress strips" (see fig. 1), to understand the implications of direction, altitude, speed and intentions of the aviators/ operators, and predict what will happen in the near future. The theory of SA is therefore well suited to understand safety-critical incidents and accidents in aviation $[6,7]$. Jones and Endsley [17] found that failing SA
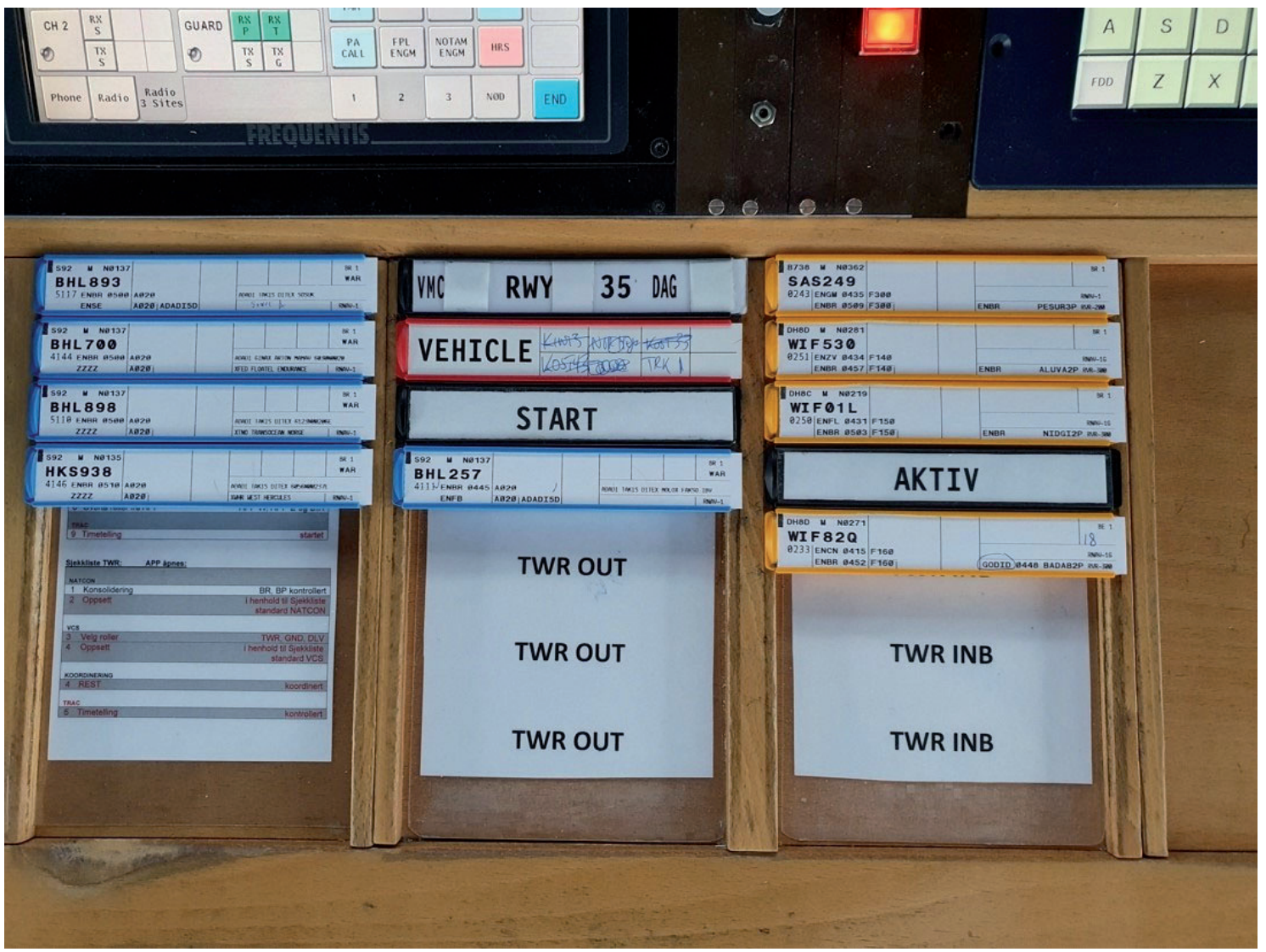

Fig. 1. Flight progress strips at one major airport in Norway. 
is a causal factor in a large proportion of aviation incidents and accidents. However, less is known about the significance of SA in runway incursion events, thus the present study will contribute to close this gap.

Air traffic control is organized into three disciplines: area control, approach control and tower control. This study focuses on tower control, which involves control of an airport and the immediate airspace around it. The air traffic tower control is responsible for the areas of the airport that the aircraft uses for take-off and landing (runways), and the taxiways the aircraft uses to get to and from the runway(s). Tower control is also responsible for aircrafts in the immediate airspace surrounding the airport. The scope of this study is to analyze all incident reports from so-called extended investigations of runway incursion incidents at Norwegian airports from 2009 to 2019 to provide a comprehensive understanding of human factor variables associated with tower control and safety critical runway incursion incidents. Our aim in the first part of this study was to identify explanatory factors from the extended investigations of seven runway incursion incidents. Our next objective was to examine if the explanatory factors from the extended inquiries could be mapped onto the theoretical framework of situational awareness (SA).

Note: Flight progress strips and flight progress board is not used in the same way across air traffic control units in Norway.

\section{METHODS}

A literature search revealed that no previous empirical studies had examined the relationship between runway incursion and SA. The explorative nature of this inquiry into a specific phenomenon called for a qualitative approach using a phenomenological hermeneutical design [4]. The reports from the extended inquiries represents the most detailed and rich empirical material from safety critical runway incidents. From a phenomenological perspective, the extended inquiries represented an opportunity to emphasize an attitude of genuine curiousness in approaching the cases.

\section{Cases}

Although minor runway incursion incidents may occur relatively frequent, the cases where the air traffic control is directly involved in a safety critical runway incursion incident resulting in an extended investigation are few. If a runway incursion has occurred, the aviation management will require an incident report. The report will be processed and assessed by dedicated safety inspectors at the aviation management in Norway. Incident reports will be followed up in different ways depending on the severity and the parties involved in the incident. Three types of followup procedures are considered when incident reports are processed. If the air navigation services are involved, the incident will be reviewed by an internal investigator [29]. These are experienced air traffic controllers and other operational staff with additional training to investigate incidents. If there is no contribution from the air navigation services, the incident report will be stored and used for statistical monitoring purposes. If the air navigation services has contributed to the incident, a desktop review will produce a report with recommendations for removing or reducing risk [29]. If the incident is considered to meet the criteria for a "serious" incident, an extended investigation will be conducted. According to Annex 13 Aircraft Accident and Incident Investigation [15] a serious incident is defined as "an incident involving circumstances indicating that there was a high probability of an accident [...] The difference between an accident and a serious incident lies only in the result".

Whereas a single internal investigator conducts a desktop review, a group of internal investigators will carry out an extended investigation. They will conduct a site visit, interview relevant individuals,

Tab. 1. Presentation of included cases and type of runway incursion (RI).

\begin{tabular}{lccccccc}
\hline Case & A & B & C & D & E & F & G \\
\hline Aerodrome & Regional & Regional & Regional & Regional & Regional & Large & Large \\
\hline Time of year & Fall & Winter & Fall & Winter & Winter & Fall & Winter \\
\hline Time of Day & Day & Day & Night & Day & Night & Night & Night \\
\hline Year & 2017 & 2016 & 2015 & 2012 & 2010 & 2009 & 2009 \\
\hline Type of RI & Vehicle & Vehicle & Vehicle & Vehicle & Vehicle & Aircraft & Aircraft \\
\hline
\end{tabular}


examine reports and assess relevant technical information. The purpose of the extended surveys is to identify underlying explanatory factors for the runway incursion event, and to provide recommendations for removing or reducing risk in a more thorough manner than in a desktop review.

For example, 135 runway incursions incidents were reported to the Norwegian Civil Aviation Authority in 2019 [3]. None of the runway incursion incidents were categorized as a serious incident with air traffic management contribution. Therefore, only desktop investigations and no extended investigations of runway incursion incidents were conducted in 2019. Due to their brief format and limited scope, desktop investigations are less suitable for qualitative content analysis. A careful examination of available records revealed that seven extended investigations had been completed in Norway from 2009 to 2019. The national air navigation services granted permission to include all seven cases in the present study.

\section{Analysis}

The examination of the case reports was carried out in several subsequent steps. The scope of extended investigations of runway incursions was first mapped and the complete reports were retrieved from the archives. To address the first research question, a directed qualitative content analysis of the case reports was performed to identify all relevant human factors variables mentioned in the inquiry. A line-by-line reading of the reports identifies human factors variables based on a process of open and axial coding [1], without adhering to an overarching typology. There has been no post-hoc analysis of causality. The human factor variables are based on the causal factors in the investigation reports. The contents of the case reports were classified according to which domain or explanatory factor it represented. For example, in one of the cases an explanatory factor was represented by the statement "air traffic controller forgot [...]", this explanatory factor was then subsequently coded in the category of "memory". The categorization was done in several rounds, where explanatory human factors units from the case reports were assessed whether they could fit into other common, or whether categories could be merged into a broader common unit [12]. After several readings and iterations this process produced four human factors categories associated with safety critical runway incursions, (see table 2 in the results section).
To address the second research question, the explanatory factors from table 2 were sorted into two main categories: «SA failure» which included explanatory factors which indicated a human error and «other» which included explanatory factors which relate to procedure design, organizational factors, or lack of equipment. Explanatory factors related to "SA failure" were further classified according to the taxonomy in Endsley [7]. In addition, examples from Jones and Endsley [17] provided helpful guidance. For example, one of the extended inquiries reads; "The air traffic controller together with [....] was busy modifying a system flight plan [...] and then forgot about the two vehicles on the runway ». According to Jones and Endsley [17] this would correspond to a level 1 error in SA since the air traffic control in this instance « [ ...] became distracted while performing job-relevant tasks and either forgot pertinent information they had previously been aware of or forgot to perform a pertinent task ». In some cases, an explanatory factor was reconsidered and reclassifies based on contextual information in the report or multiple iterations and comparison of classification across cases. For example, the explanatory factor «runway incursion alarm worked normally, but was turned off » was initially considered to represent a missing, monitoring or observation of data. Later this factor was re-categorized as data not available since nothing in the situation prevented or distracted the air traffic control from hearing the alarm if the system had been activated in the first place, (see table 3 in the results section).

\section{Ethics}

Access to extended investigation reports were granted by the national air navigation services on condition that records were kept confidential and the identity of involved personnel was not disclosed [9]. Detailed information that could identify individuals, customers or specific locations is therefore not disclosed. The second author is an air traffic controller and supervisor. None of the incidents took place at a unit where he worked nor did he have any personal relations to any of the subjects mentioned in the extended investigations.

\section{RESULTS}

The reports from the extended investigations describe what the investigators see as primary human factors causes and/or contributing reasons why the safety critical runway incursions occurred. These causes, whether they are stated as a conclu- 
Eid J. et al. - Runway incursions...

Tab. 2. Main categories extracted from seven extended investigations of runway incursion incidents in Norway from 2009 to 2019.

\begin{tabular}{|c|c|c|c|c|c|c|c|}
\hline Main categories & Case & & & & & & \\
\hline & A & B & $C$ & D & $\mathrm{E}$ & $\mathrm{F}$ & G \\
\hline Perception & 6 & 3 & 2 & 4 & 4 & & 2 \\
\hline $\begin{array}{l}\text { Procedural } \\
\text { errors }\end{array}$ & 3 & 3 & 4 & & 1 & & 2 \\
\hline Memory & 1 & 1 & 1 & 1 & & & \\
\hline Decision-making & & & & & 1 & 1 & 2 \\
\hline
\end{tabular}

Note: Each report can contain several explanatory factors from different categories. Event A, for example, has 10 explanatory factors discussed in the report, while event $F$ has only one. Explanatory factors $(n=42)$ are indicated by numbers associated with each event. Each of the seven extended investigation (case) reports are represented by the letters $A$ to $G$.

sive or most likely cause in the report were identified as explanatory factors. In total 42 explanatory factors were identified across the seven reports. After a careful review and repeated processing, these 42 factors were condensed into four categories; perception, procedural errors, memory, and decision-making, (see table 2). In the following, these categories will be further elaborated with examples from the reports.

Explanatory factors related to perception were associated with safety critical runway incursion in six of the seven cases. These six extended studies contained 21 different examples of explanatory factors related to perception. In total, factors related to perception accounted for $50 \%$ of the total number of explanatory factors across all cases. The explanatory factors related to perception described deficits and problems related to visual perception, auditory perception and attention. Examples of explanatory factors related to visual perception were "warning lights that were difficult to see in daylight" and "obstructed view prevented the air traffic controller from seeing the vehicle on the runway". Explanatory factors related to auditory perception included for example that aircraft and vehicles operated on different radio frequencies within the same parameter. Other examples were related to competing auditory information. Explanatory factors related to attention were, for example, a case where the air traffic controller may have been distracted from the primary task; "the air traffic controller together with the air traffic control assistant was busy changing a system flight plan [...]". Reduced vigilance was also seen to influence attention; "There are several registered incidents that have occurred in the period after a traffic peak with a high workload. Incidents can occur because of the concentration decreasing when the traffic and load decrease".

In five of seven extended studies human factor issues relating to procedural errors were reported 13 times as explanatory factors associated with safety critical runway incursion. The explanatory factors in these five reports emphasized that procedures had been used infrequently, incorrectly, not used at all, or that the procedures themselves had shortcomings. Issues related to procedures accounted for $31 \%$ of the total number of explanatory factors across cases. In two cases failure to adhere to procedures or deactivation of procedures had resulted in a safety critical runway incursion. In another case, a runway incursion alarm-system was disabled to avoid false alarms. This was in accordance with the standard operating procedures, but the investigation considered this a contributing factor to why the runway incursion was not detected earlier. Finally, inadequate procedures or unclear communication when handing over the maneuvering area and inadequate reporting of positions of vehicles are also noted as explanatory factors related to procedural issues in two of the reports.

In four of the seven extended studies human factor issues relating to memory were reported as explanatory factors associated with safety critical runway incursion. In all incidents where an explanatory factor involved memory the air traffic controller had forgotten that there was a vehicle on the runway. Issues related to memory accounted for $10 \%$ of the total number of explanatory factors across cases.

In three of the seven extended studies human factor issues relating to decision making were reported four times as explanatory factors associated with safety critical runway incursion. These situations involved decisions that lead to safety critical situations at the runway, caused by the air traffic controller or other individuals at the airport. Issues related to decision making accounted for $10 \%$ of the total number of explanatory factors across cases.

\section{Explanatory human factor variables and situation awareness (SA)}

Our next objective was to examine if the explanatory factors from the extended inquiries 
Tab. 3. Classification of explanatory factors in extended inquiries according to SA theory.

\begin{tabular}{|c|c|c|}
\hline Level of error & No. of explanatory factors & Case \\
\hline Data not available & 7 & $A B C G$ \\
\hline Hard to discriminate or detect data & 9 & $A D E G$ \\
\hline Failure to monitor or observe data & 3 & BEG \\
\hline Misperception of data & 1 & $C$ \\
\hline Total level 1 error & 24 & \\
\hline \multicolumn{3}{|c|}{ Level 2: Improper integration or comprehension of information } \\
\hline Lack of or incomplete mental model & 2 & CG \\
\hline Use of incorrect mental model & 0 & \\
\hline \multicolumn{3}{|c|}{ Level 3: Incorrect projection of future actions of the system } \\
\hline Lack of or incomplete mental model & 0 & \\
\hline Over projection of current trends & 0 & \\
\hline Other & 2 & CF \\
\hline Total level 3 errors & 2 & \\
\hline Total errors & 30 & \\
\hline
\end{tabular}

Note: Based on taxonomy from Endsley [7] and Jones and Endsley [14].

could be mapped onto the theoretical framework of $S A$ and provide a more comprehensive understanding of human factor variables associated with safety critical runway incursions. According to the classification taxonomy from Endsley [7], errors in situational awareness were evident in all the seven extended investigations of safety critical runway incursion events. In total, 30 of the 42 explanatory factors could be attributed to errors due to failure in SA. The remaining 12 explanatory factors were primarily related to non-human factor shortcomings (i.e., insufficient equipment, weather conditions, lack of procedures or procedural design).

According to the results presented above, 30 explanatory factors could be associated with SA failure, $80 \%(n=24)$ indicated a level one error of failure in perception, $13 \%(n=4)$ indicated a level two error of failure to comprehend the situation and $7 \%(n=2)$ indicated a level three error of failure to project future actions. Six of these explanatory factors could be attributed to external subjects, while 24 could be attributed to the air traffic controller, trainee, or instructor. On the job training took place in two of the seven incidents.

Failure in perception or misconception of information (level one SA) was the most frequent explanatory factor in the extended investigations. In total 24 out of 30 explanatory factors indicated a level one error in SA where information was not available, was difficult to detect, comprehend or remember. In the category information not available, there were seven explanatory factors from four different events. Four explanatory factors were related to flight progress strips, which reminded the air traffic controller where the aircraft and vehicle were $(A, B, C)$. Two explanatory factors were related to functionality that was disabled or not enabled to provide a reminder of vehicles on the runway $(A, B)$. One explanatory factor was related to the runway incursion alert system which was disabled to avoid false alarms (G). The largest subcategory of failure in perception was difficult to discriminate between, or detect information, with nine explanatory factors divided into four reports $(A, D, E, G)$. For instance, there was a vehicle on a part of the runway that was not visible from the tower. Flight progress strips which marked vehicles on the runway contained information that there were two vehicles on the runway, but only one was instructed to leave and a warning light indicating that there were still vehicle(s) on the runway were not detected $(D)$. There were three explanatory factors that could be classified as lack of monitoring or observation of information. These factors were mentioned in three reports $(B$, E, G). Among other things, an explanatory factor about the air traffic controller's attention, which was directed at editing a flight, plan together with the air traffic control assistant, and not the runway. Only one explanatory factor could be classified as misconception of information. This is an ex- 
planatory factor where a position report leads to a misunderstanding about a vehicle's position. In four reports, there were four different cases of explanatory factors that could be classified as failing memory. All cases involved explanatory factors where the air traffic controller forgot that there was a vehicle on the runway.

Level two SA requires that information not only needs to be perceived, but also must be understood and integrated with other pieces of information. Four of the explanatory factors indicated a failure to integrate or understanding available information. Missing or incomplete mental models were mentioned in two of the cases. Example: An aircraft crosses a lit stop bar with clearance from the air traffic controller, but the regulations give the stop light row priority over the air traffic controller's clearance which could indicate that the crew's mental model of traffic near the stop bar was not complete (G). Stop bars are intended to be an additional barrier if an aircraft mistakenly thinks they are receiving a clearance intended for another aircraft. The stop bar must have been clearly visible. Excessive reliance on default values was found in two explanatory factors in two different reports. Default values can be considered as expectations of how the system works in the absence of real-time data. Both explanatory factors were related to slips or lapses. An example was an explanatory factor where the air traffic controller issued a "line up" clearance to a runway he had cleared another aircraft to land (G).

A level three error in SA indicates a failure to project future events. Two of the cases indicated that the air traffic controller had a missing or incomplete mental model for projection of future status in the system. An example of this was a situation where the air traffic controller misjudged the spacing between two landing aircraft and executed a departure between them whereby not securing appropriate spacing between the departure and the second landing.

\section{DISCUSSION}

The purpose of this study was to identify explanatory factors in extended investigations of critical RI incidents in Norway and to examine if situational awareness (SA) could contribute to a more comprehensive understanding human factors in runway incursion incidents. Avinor Air Navigation Services' procedures describe how incidents are treated and how investigations should be carried out [29]. The reports from the extended investigations vary somewhat in scope due to the complexity of the event, but they all follow the same template. The seven available reports represented all available reports from the most recent decade of aviation safety in Norway and provided a comprehensive empirical basis for the study.

The results from the directed qualitative content analysis of the seven reports show that the causal relationships of the extended investigations are complex and context dependent. The explanatory factors highlight the significance of human factors such as behavior, cognition or inherent risk factors related to organization, technology, or environmental issues. The present study contributes to this end by identifying four human factor categories related to the extended investigation of runway incursion s over the last decade in Norway. These factors are perception, procedural errors, memory, and decision-making.

The extended investigation reports present a complex picture of cognitive, operational and contextual factors associated with the dynamic nature of air traffic control. This is consistent with previous research that has described inherent hazards in complex casual chains of operations in safety critical organizations and the need to maintain multiple safety barriers [26]. The group of internal investigators also expresses that it proved difficult to find a simple explanatory factor in events $F$ and $G$. It can be challenging to identify human factors if one does not link it to a specific typology or method for identifying such factors.

Perception. In total, 21 out of 42 explanatory factors belong to the category of perception. The category includes all explanatory factors where perception and attention may have been involved. Four of the 42 explanatory factors are related to memory, and four are related to decisionmaking. It is widely accepted that the cognitive factors of perception, attention and memory are closely interrelated [24]. In a study of simulated air traffic control environments, researchers found that audible alarms were ignored in between $28-48 \%$ of cases [10]. The study suggested that competing sensory information could inhibit attention to new and unexpected auditory stimuli. In event $G$, there are several auditory stimuli competing for attention. The air traffic controller receives an incoming telephone call at the same time as an aircraft calls on the VHF radio and an auditory alarm warns about a runway incursion incident where an aircraft is crossing a lit stop bar. The extended investigations indicate that not only auditory stimuli may remain undetected. Prominent visual stimuli and changes in the visual field can also be overlooked [22]. Expectancy bias, 
perceptual cues and goals can influence attention and what one visually searches for [2]. This phenomenon may have occurred in incident $D$. The air traffic controller asks one of two vehicles to leave the runway and then performs a visual inspection before he clears an aircraft for departure even if there is still one vehicle left on the runway. The air traffic controller apparently overlooked or potentially did not expect to see more vehicles on the runway before he gave permission for departure. Also, in incident $D$, the second vehicle was only visible via video surveillance from a part of the runway which was not visible from the tower. Several factors affect working memory [11], including individual differences, but also contextual factors. Chronic stress and high workload are examples of factors that can negatively affect working memory. However, acute stress can promote memory [11]. There is good empirical support for the fact that limitations in memory are closely linked to limitations in attention [24]. This relationship may indicate that the explanatory factors associated with memory may be related to a limited capacity of attention and not the coding between working memory and long-term memory.

Perception, attention, and memory all play a role in decision making. One often distinguishes between different types of decision-making such as analytical decision-making and intuitive decision-making [19]. In the explanatory factors that deal with decision-making, it is first and foremost factors around intuitive decision-making that are important. Experts can quickly make decisions according to principles of recognition-primed decision-making [21]. Although intuitive decisionmaking is an advantage in operational situations, it is also vulnerable to biases or 'bias' [20]. In incident $F$, the air traffic controllers choose to continue his plan by not canceling a departure in front of a landing, even if the available information could indicate that the plan should have been changed. Orasanu, Ames, Martin, and Davison [25] call this a "plan continuation" error. They suggest that this type of error can occur based on stress, cognitive factors, contextual factors, or from social or organizational pressure.

Procedures. Out of 42 explanatory factors, 13 belong to the category of procedures. These are explanatory factors where a procedure has not been followed, the procedure has weaknesses, procedures are missing, or the procedure is rarely used. In events $A$ and $B$, none of the methods for marking vehicles on the runway are used. Both events take place at the same airport. In case $A$, the group of internal investigators writes that there are frequent and rapid runway crossings at this airport where the use of "busy runway"-markings seldom are used. According to SKYbrary [28], there may be several factors behind non-compliance to procedures. This includes inadequate procedures, social and organizational pressure, convenience, and professional arrogance. It may also be that this is due to habituation or instrumental conditioning since this is often repeated without consequences, which may reduce the use of these procedures. One way to prevent this is to introduce false alarms to disabling the behavior, but this must not be done too often as this can be habituated [16].

Our final objective in this study was to examine if the explanatory factors from the extended inquiries could be mapped onto the theoretical framework of situational awareness (SA). Our results indicate that failure to comprehend the situation emerged as a significant outcome across all seven extended inquiries. The present study is in line with previous research from Endsley [7] in that 30 out of 42 explanatory factors could be related to failures in SA. Compared to Jones and Endsley [17] our results follow the same trend in that $80 \%$ (compared to $76.3 \%$ ) could be attributed to level one, failure in perception. Furthermore, 13\% (compared to $20.3 \%$ ) could be attributed to level two, failure to comprehend the situation and finally $7 \%$ (compared to $3.4 \%$ ) could be attributed to level three, failure to project future events. The main source of failure in SA emerges at the first level, failure in perception. The relatively low proportion of errors at the second and third level may be because it is difficult to determine that there is in fact an error at levels two and three based on incident reports [17], but also that an error is primarily identified at the lowest possible level. An error at the first level will probably also lead to errors at the second and third levels. Where errors at levels two and three are often related to experience and training, errors at the first level may be based on the design of the work position and help systems, but it may also be related to human cognitive and biological limitations.

Taken together, our findings are in line with previous research and suggests that the theory of SA may present a meaningful way to organize retrospective analyzes of runway incursion events to obtain a more holistic picture of system-critical human factor variables. The theory of SA could serve as a useful starting point for understanding the connection between the various explanatory factors. For example, the reports from events $A$ and $B$ have different conclusions, but several 
of the same explanatory factors are present. Although the events themselves were different, it gives a better understanding of the system-critical human factors when one considers them as a failure in SA.

Some notable limitations should be observed. Although failures related to SA emerged as a significant factor across all cases, it is not possible to determine why SA failed at the case level based on the accident reports. An exclusive emphasis on cognitive factors related to SA may risk missing the fact that this is part of a larger a whole. There are explanatory factors that do not imply failure in SA in most reports, but most explanatory factors imply failure in SA. The extended inquiries also pointed to explanatory factors that are not directly related to human factors such as insufficient equipment or weather conditions that needs to be considered in investigation of runway incursion incidents. The present study is based on relatively few $(\mathrm{N}=7)$ cases. Although a larger sample could have produced a more diverse material, the selection of cases includes all available extended investigations from the last decade and therefore represents a quite representative sample from the Norwegian aviation sector.

\section{CONCLUSIONS}

The present study provides a comprehensive assessment and classification of all runway incursion incidents in Norway from the last decade. The present study provides empirical support to the significance of attending to human factor variables and in particular cognitive factors associated with perception, memory, decision-making and procedural errors. In accordance with previous research, our findings indicates that the concept of situational awareness is an important human factor variable in assessing and training of air traffic controllers $[18,27,30]$. Such training could be both psychoeducation about the cognitive elements of situational awareness and in true-to life simulator exercises. Support systems and procedures used by air traffic controllers should be critically evaluated to determine whether they improve SA or not.

Although our study suggests that the theoretical concept of situational awareness contributes to our understanding of safety critical human factor elements in aviation, this perspective has also been criticized by Dekker and Hollnagel [5] for not considering distributed cognition and more specifically human-computer interaction in air traffic control. Traditionally, the human user has mainly been conceived as a relatively passive recipient of fused information. More recently, the importance of understanding the active role of human information processing in information fusion is gaining increasing recognition [23]. Future research on aviation safety could therefore benefit from a more detailed analysis of cognitive processes and information flows involved in how air traffic controllers and decision support systems collaboratively contribute to aviation safety and preventions of runway incursions [27]. Experimental studies in air traffic control simulators could unpack the dynamic nature of information fusion in aviation and how this could contribute to future aviation safety.

\section{AUTHORS' DECLARATION:}

Concept: Jarle Eid, Preben Bjarkøy Jensen. Development of assumptions: Jarle Eid, Preben Bjarkøy Jensen. Graphic design and text editing: Jarle Eid, Preben Bjarkøy Jensen. The Authors declare that there is no conflict of interest.

\section{REFERENCES}

1. Bernard HR. Social research methods: Qualitative and quantitative approaches. Sage Publikations, Thousand Oaks, CA, USA, 2013.

2. Biggs AT, Adamo SH, Dowd EW, Mitroff SR. (2015). Examining perceptual and conceptual set biases in multiple-target visual search. Attention, Perception \& Psychophysics, 2015; 77(3): 844-855. doi: 10.3758/s13414-014-0822-0.

3. CAA Norway. Flysikkerhetsinformasjon: Runway incursion, 2020. Retrived from: http://pdf.caa.no/ri.pdf.

4. Creswell JW. Qualitative Inquiry and Research Design: Choosing Among Five Traditions, 2nd ed.; Sage Publications, Thousand Oaks, CA, USA, 2007.

5. Dekker S, Hollnagel E. Human factors and folk models. Cognition, Technology \& Work, 2004; 6(2): 79-86.

6. Endsley MR. Toward a theory of situation awareness in dynamic-systems. Human Factors, 1995a; 37(1): 32-64. doi: 10.1518/001872095779049543. 
7. Endsley MR. A taxonomy of situation awareness errors. Human factors in aviation operations, 1995b; 3(2): $287-292$.

8. Eurocontrol. European action plan for the prevention of runway incursions v3.0, 2017. Retrived from: https://www.eurocontrol. int/sites/default/files/2019-06/european-action-plan-prevention-runway-incursions-v3.pdf.

9. Freedom of Information Act, 2006, §14. Act relating to the right of access to documents held by public authorities and public undertakings (LOV-2006-05-19-16). Retrived from: https://lovdata.no/NLE/lov/2006-05-19-16.

10. Giraudet L, Imbert JP, Tremblay S, Causse M. High Rate of Inattentional Deafness in Simulated Air Traffic Control Tasks. Procedia Manufacturing, 2015; 3:5169-5175. doi: 10.1016/j.promfg.2015.07.555.

11. Hansen AL. Hukommelse [Memory]. In Eid J, Johnsen BH. (Eds.), Operativ psykologi (2nd ed.). Bergen: Fagbokforl, 2006; 65-79.

12. Hsieh HF, Shannon SE. Three Approaches to Qualitative Content Analysis. Qualitative Health Research, 2005; 15(9): 12771288. doi: 10.1177/1049732305276687.

13. International Civil Aviation Organization. Procedures for Air Navigation Services (PANS) - Air Traffic Management (Doc 4444). Montreal: International Civil Aviation Organization 2016.

14. International Civil Aviation Organization. Annex 11 Air traffic Services [Ebook], 15th ed., 2018, pp. 1-3.

15. International Civil Aviation Organization. Annex 13 Annex 13 Aircraft Accident and Incident Investigation [Ebook], 11th ed., 2016: 1-3.

16. Johnsen BH. Læringspsykologi [Behavioral psychology]. In Eid J, Johnsen BH. (Eds.), Operativ psykologi (2nd ed.). Bergen: Fagbokforl, 2006; 51-64.

17. Jones DG, Endsley MR. Sources of situation awareness errors in aviation. Aviation, space, and environmental medicine, 1996: 507.

18. Jones DR. Runway incursion prevention system simulation evaluation. In Proceedings. The 21st Digital Avionics Systems Conference. IEEE, 2002; 2: 11B4-11B4.

19. Kahneman D. Thinking, fast and slow Macmillan, 2011.

20. Kahneman, D, Slovic P, Tversky A. Judgment under uncertainty: Heuristics and biases Cambridge university press, 1982.

21. Klein GA. A recognition-primed decision (RPD) model of rapid decision making. Decision making in action: Models and methods. 1993; 5(4): 138-147.

22. Matlin MW. Cognitive psychology (8th ed. International student version. utg.). Hoboken, N.J: Wiley, 2014.

23. Nilsson M, van Laere J, Susi T, Ziemke T. Information fusion in practice: A distributed cognition perspective on the active role of users. Information fusion, 2012; 13(1): 60-78.

24. Oberauer K. Working Memory and Attention-A Conceptual Analysis and Review. Journal of cognition, 2019; 2(1).

25. Orasanu J, Ames N, Martin L, Davison J. Factors in aviation accidents: decision errors. Linking expertise and naturalistic decision-making, 2001: 209.

26. Reason, J., Hollnagel, E. \& Paries, J. Revisiting the Swiss cheese model of accidents. Journal of Clinical Engineering, 2006; 27(4): 110-115.

27. Schönefeld J, Möller DPF. Runway incursion prevention systems: A review of runway incursion avoidance and alerting system approaches. Progress in Aerospace Sciences, 2012; 51: 31-49.

28. SKYbrary. (30.09.2010). Non-compliance with ATC Procedures in low traffic periods, 2005. Retrived from: https://www. skybrary.aero/index.php/Non-compliance_with_ATC_Procedures_in_low_traffic_periods.

29. Tellevik BEU. FS-P-S031 Behandling av luftfartshendelser og -ulykker i Avinor Flysikring AS. [Assessment of aviation incidents and accidents in Avinor Air Navigation Services]. Avinor Governing Documents Appendix B. 2018.

30. Vernaleken C, Urvoy C, Klingauf U. Prevention of runway incursions due to closed runways or unsuitable runway choices by enhanced crew situational awareness and alerting. In Enhanced and Synthetic Vision, 2007; 6559: 65590I.

31. Wickens CD. Situation Awareness: Review of Mica Endsley's 1995 Articles on Situation Awareness Theory and Measurement. Human Factors, 2008; 50(3): 397-403. doi: 10.1518/001872008x288420.

Cite this article as: Eid J., Jensen P. B. Runway Incursions In Norway 2009-2019: A Case Study Of Extended Investigation Reports. Pol J Aviat Med Bioeng Psychol 2019; 25(3): 5-14. DOI: 10.13174/pjambp.19.05.2021.01 